\title{
The effects of vitamin A supplementation with measles vaccine on leucocyte counts and in vitro cytokine production
}

\author{
Kristoffer Jarlov Jensen ${ }^{1,2,3 * *+, ~ A n e ~ B æ r e n t ~ F i s k e r ~}{ }^{1,2}$, Andreas Andersen ${ }^{2}$, Erliyani Sartono ${ }^{4}$, \\ Maria Yazdanbakhsh ${ }^{4}$, Peter Aaby ${ }^{1,2}$, Christian Erikstrup ${ }^{5}$ and Christine Stabell Benn ${ }^{1,2,6}$ \\ ${ }^{1}$ Bandim Health Project, INDEPTH Network, Apartado 861, Bissau Codex 1004, Bissau, Guinea-Bissau \\ ${ }^{2}$ Research Center for Vitamins and Vaccines, Bandim Health Project, Statens Serum Institut, Artillerivej 5, DK-2300 \\ Copenhagen S, Denmark \\ ${ }^{3}$ Section for Immunology and Vaccinology, National Veterinary Institute, Technical University of Denmark, Bülowsvej 27, \\ DK-1870 Frederiksberg C, Denmark \\ ${ }^{4}$ Leiden University Medical Center, Gebouw 1, Kamer P4-25a, Postbus 9600, 2300 RC Leiden, The Netherlands \\ ${ }^{5}$ Department of Clinical Immunology, Aarbus University Hospital, Palle Juul-Jensens Boulevard 99, DK-8200 Aarbus, \\ Denmark \\ ${ }^{6}$ Odense Patient data Explorative Network (OPEN), Department of Clinical Research, Odense University Hospital, J.B. Winsløws \\ Vej 9A, 3, DK-5000 Odense C, Denmark
}

(Final revision received 10 June 2015 - Submitted 26 October 2015 - Accepted 4 November 2015 - First published online 18 December 2015)

\section{Abstract}

As WHO recommends vitamin A supplementation (VAS) at vaccination contacts after age 6 months, many children receive VAS together with measles vaccine (MV). We aimed to investigate the immunological effect of VAS given with MV. Within a randomised placebo-controlled trial investigating the effect on overall mortality of providing VAS with vaccines in Guinea-Bissau, we conducted an immunological sub-study of VAS $v$. placebo with MV, analysing leucocyte counts, whole blood in vitro cytokine production, vitamin A status and concentration of C-reactive protein (CRP). VAS compared with placebo was associated with an increased frequency of CRP $\geq 5 \mathrm{mg} / 1(28 v .12 \% ; P=0 \cdot 005)$. Six weeks after supplementation, VAS had significant sex-differential effects on leucocyte, lymphocyte, monocyte and basophil cell counts, decreasing them in males but increasing them in females. Mainly in females, the effect of VAS on cytokine responses differed by previous VAS: in previous VAS recipients, VAS increased the pro-inflammatory and T helper cell type 1 (Th1) cytokine responses, whereas VAS decreased these responses in previously unsupplemented children. In previous VAS recipients, VAS was associated with increased IFN- $\gamma$ responses to phytohaemagglutinin in females (geometric mean ratio (GMR): 3.97; $95 \%$ CI 1.44, 10.90) but not in males (GMR 0.44; $95 \%$ CI $0.14,1.42)$; the opposite was observed in previously unsupplemented children. Our results corroborate that VAS provided with MV has immunological effects, which may depend on sex and previous VAS. VAS may increase the number of leucocytes, but also repress both the innate and lymphocyte-derived cytokine responses in females, whereas this repression may be opposite if the females have previously received VAS.

Key words: Vitamin A supplementation: Paediatric nutrition: Cytokines: Differential count: Heterologous immunity: Inflammation

Vitamin A is important for fundamental immunological functions, including modulation of differentiation, maturation, migration or activation of dendritic cells, T cells and NK cells ${ }^{(1)}$.

The WHO estimates that 190 million children under the age of 5 years worldwide are at risk of vitamin A deficiency (VAD), especially in Africa and South East Asia ${ }^{(2)}$. To alleviate and prevent VAD, the WHO recommends high-dose vitamin A supplementation (VAS) at routine vaccination contacts after 6 months of age ${ }^{(3)}$. According to the current vaccination schedule, the first vaccine to be given after the age of 6 months is the measles vaccine (MV) scheduled at age 9 months ${ }^{(4)}$.

Vaccines may have effects on overall mortality and morbidity beyond the specific protection against the targeted disease - the so-called non-specific effects ${ }^{(5)}$. Many studies have shown that live attenuated MV has beneficial effects on mortality far exceeding what can be ascribed to the prevention of measles cases, presumably due to immune-modulating effects, leading to increased resistance towards unrelated infections ${ }^{(5-7)}$.

Abbreviations: CRP, C-reactive protein; GMR, geometric mean ratio; MV, measles vaccine; PHA, phytohaemagglutinin; PR, proportion ratio; RBP, retinol-binding protein; VAD, vitamin A deficiency; VAS, vitamin A supplementation.

* Corresponding author: K. J. Jensen, email kjj@ssi.dk

$\dagger$ Data and study protocol are available upon request to the corresponding author. 
Several studies have suggested that high-dose VAS and vaccines interact with effects on overall mortality and morbidity. Our group has previously found that VAS together with MV increases the MV-specific antibody response ${ }^{(8,9)}$. We have hypothesised that VAS may enhance both the measles-specific and the non-specific effects of MV via beneficial general immune-stimulating effects ${ }^{(10)}$.

In Guinea-Bissau, from 2007 to 2011, before the implementation of the WHO policy of giving VAS at vaccination contacts, we conducted a randomised placebo-controlled trial to evaluate the effect of this recommendation. At vaccination contacts after 6 months of age, children were randomised to VAS or placebo, the main outcomes being all-cause child mortality and morbidity.

Nested within the trial, we performed a sub-study to investigate the effects of VAS together with MV on non-measlesspecific immunological outcomes. At baseline and 6 weeks after randomisation, we measured vitamin A levels, C-reactive protein (CRP) levels, differential counts and in vitro cytokine production to allow simultaneous evaluation of the effect of VAS on different parts of the immune system.

Prompted by previous studies showing that the effect of VAS on morbidity or mortality depends on $\operatorname{sex}^{(11,12)}$, season of supplementation ${ }^{(13)}$ or previous high-dose $\operatorname{VAS}^{(14)}$, we pre-specified to investigate the possible interactions between VAS, sex, season of supplementation and previous VAS. Indeed, the main trial corroborated that there are important interactions: there was no overall mortality effect of VAS, but the effect was sex-differential, with VAS being beneficial for females but detrimental for males. These sex-differential effects were most evident in the dry season and in children who had previously received VAS $^{(15)}$.

\section{Methods}

The present immunological sub-study was nested within a randomised trial of administering VAS $v$. placebo together with routine vaccinations in Guinea-Bissau, described in detail elsewhere $^{(15)}$.

\section{Main trial}

Between August 2007 and November 2010, children aged between 6 and 23 months were invited to participate in the main trial when they came to the healthcare centres for routine vaccination. A study nurse inspected the vaccination cards and identified eligible children. VAS within the preceding month was an exclusion criterion. If parents provided oral and written consent, the children were randomised to vitamin A or placebo groups. Randomisation was stratified by child sex. Mothers drew a lot from an envelope containing twenty lots; ten for each treatment arm. The number on the lot indicated from which of the two numbered bottles the children would receive an oral supplement. Children under the age of 1 year received $0.5 \mathrm{ml}$; children who had reached 1 year of age received $1 \mathrm{ml}$. For the children in the VAS group, this meant that they received $100000 / 200000 \mathrm{IU}$ vitamin A (retinyl palmitate) dissolved in vegetable oil with vitamin $\mathrm{E}$ added as an antioxidant $(40 \mathrm{IU} / \mathrm{ml})$.
Children in the placebo group received the same amount of oil with vitamin E. MV was supplied by $\mathrm{UNICEF}^{(15)}$. National VAS campaigns were conducted by the Ministry of Health approximately every 6 months. One month before the announced date of a campaign, inclusion into the main trial was paused in order to prevent children from receiving two doses of VAS within a month ${ }^{(16)}$. In the month following a campaign, we included only children who had not received VAS in the campaign.

\section{The immunological sub-study}

Between September 2007 and June 2009, children who were due to receive $\mathrm{MV}$ were additionally invited to participate in the immunological sub-study. The mothers were explained that participation was voluntary and that participation in the main study was not contingent upon enrolment in the sub-study. Oral and written consent was obtained separately for the sub-study.

With consent, a blood sample was obtained before randomisation in the main trial to vitamin A or placebo. All children from whom we had obtained a blood sample at inclusion were visited by a field assistant 6 weeks after inclusion and were asked to visit the healthcare centres for the follow-up blood sample collection. If the child could not be found at home, the assistant kept revisiting the home until $100 \mathrm{~d}$ had passed since inclusion; the child was then registered as lost to follow-up.

\section{Blood sampling procedures and analyses}

Before blood sampling, axillary temperature, weight and mid-upper arm circumference (MUAC) were measured, and the mother was interviewed about the health of the child, including symptoms of disease on the day of blood draw and use of medicines during the $3 \mathrm{~d}$ before blood draw. Venous blood was drawn into a heparinised tube and an EDTA tube. The EDTA tubes were kept in a cool box until arrival at the laboratory where they were left at ambient temperature to acclimatise before measurement. The heparinised blood was maintained at ambient temperature. A thick and thin blood slide was prepared for examination of malaria. The slides were Giemsa-stained and microscopically examined for malaria parasites by an experienced laboratory technician. All mothers were informed of the results of the malaria slide by a visiting field assistant on the following day. None of the children had positive malaria slides.

\section{Leucocyte counts}

Automated differential counts were performed on blood collected in EDTA tubes using an ABX pentra 60 (HORIBA $\mathrm{ABX}$ ). The accuracy and precision of measurements were validated at regular intervals using a commercial standard preparation (ABX Difftrol; HORIBA ABX). After the analyses, the EDTA tubes were centrifuged to separate plasma.

\section{Retinol-binding protein and C-reactive protein}

Plasma was analysed for retinol-binding protein (RBP) and CRP. RBP and CRP were analysed using sandwich ELISA as described elsewhere ${ }^{(17)}$, except that the poly-clonal chicken anti-human RBP secondary antibody was from Immunology Consultants Laboratory (cat. no: CRBP-80P-Z). Before measurements, we 
validated the performance of the antibody against the originally used antibody in collaboration with the developer of the assay (Jürgen Erhardt). Samples were measured in duplicate and re-measured if the CV between duplicates was above $20 \%$ for RBP and above $20 \%$ for samples with a mean value between 3 and $7 \mathrm{mg} / 1$ for CRP.

\section{Whole blood stimulation assay}

The heparinised blood was maintained at ambient temperature but shielded from direct sunlight until processing. The blood was diluted 1:10 with RPMI-1640 medium without glutamine with added streptomycin $(100 \mu \mathrm{g} / \mathrm{ml})$, penicillin A $(100 \mathrm{IU} / \mathrm{ml})$, glutamate ( $2 \mathrm{~mm}$ ) and pyruvate (1 mm) (all from Invitrogen). Stimulations were performed in sterile multiwell plates (Nunclon U-shape; NUNC) in a final volume of $200 \mu \mathrm{l}$ using phytohaemagglutinin (PHA, $2 \mu \mathrm{g} / \mathrm{ml}$; Wellcome Diagnostics), Lipopolysaccharide (LPS, $1 \mathrm{ng} / \mathrm{ml}$; Sigma-Aldrich Chemie), Polyinosine-polycytidylic acid (Poly I:C, $10 \mu \mathrm{g} / \mathrm{ml}$; InvivoGen), palmitoyl(3)-cysteine-serine-lysine(4) (Pam3cys, $100 \mathrm{ng} / \mathrm{ml}$; InvivoGen), purified protein derivative of Mycobacterium tuberculosis (PPD, $10 \mu \mathrm{g} / \mathrm{ml}$; Statens Serum Institut), tetanus toxoid (TT, 1.5 Lf/ml; Statens Serum Institut), Bacille CalmetteGuérin vaccine (BCG, 1:1000 of vaccine preparation; Statens Serum Institut), oral polio vaccine (OPV, 1:100 dilution of vaccine preparation; GlaxoSmithKline Biologicals) and diphtheria toxoid (DT, $1 \mathrm{Lf} / \mathrm{ml}$; Statens Serum Institut). Medium alone samples (medium) were included as well. Culture plates were incubated at $37^{\circ} \mathrm{C} / 5 \% \mathrm{CO}_{2} / 100 \%$ humidity. Supernatants were collected on day 1 (medium1, LPS and Pam3cys) or on day 3 (medium3, PHA, Poly I:C, BCG, PPD, OPV, TT and DT) and maintained below $-40^{\circ} \mathrm{C}$ until analysis. Each culture well was visually assessed upon harvest for signs of contamination.

Supernatant concentrations (the lower limit of detection (LLD) of the assay in $\mathrm{pg} / \mathrm{ml}$ is given in parenthesis) of IL-2 (6), IL-10 (5), IL-5 (3), IFN- $\gamma$ (5) and TNF- $\alpha$ (10) were measured simultaneously using a Luminex cytokine kit (Luminex Corporation) and a buffer reagent kit (BioSource) on a Luminex-100 cytometer (Luminex Corporation), equipped with STarStation software (Applied Cytometry Systems). The samples were tested in a random order (VAS and placebo mixed), but with paired baseline and follow-up samples from one subject in the same assay.

\section{Sample size considerations}

In previous studies utilising a similar setup, we had observed significant differences in cytokine production using samples sizes of approximately 200 children in each group in a cross-sectional design. In the present study, we included baseline samples to reduce the intra-individual noise and the necessary sample size accordingly. With an expected $20 \%$ loss to follow-up, we aimed to include 125 children per group - that is, 100 children per group for the final analyses.

\section{Statistical analysis}

Analyses were performed using Stata 12 (StataCorp LP). Cytokine data were log transformed. For several of the cytokine outcomes, a proportion of the measurements was below the LLD of the assay, defined by the manufacturer (see above), and denoted here as non-detectable (ND) measurements. ND measurements were handled by multiple imputations based on Tobit regression $^{(18,19)}$. The parameters of the underlying normal distribution are estimated by Tobit regression and values below the detection limit are drawn multiple times from a truncated normal distribution. The imputations incorporate the correlation between baseline and follow-up samples. Geometric mean ratios (GMR) were obtained as anti-logged coefficients. For distributions with $>50 \%$ ND measurements, the proportion of detectable samples was analysed by Poisson regression giving proportion ratios (PR).

Where all the measurements in a subgroup analysis were below LLD in either of the randomisation groups, Poisson regression was not possible, and CI were obtained using profile-log-likelihood estimation ${ }^{(20)}$.

For distributions with $<50 \%$ ND measurements, the ratios of IFN- $\gamma:$ IL- 5 and TNF- $\alpha$ :IL-10 responses were analysed as crude markers of the balance of Th1 $v$. Th2 cytokine responses and the pro- $v$. anti-inflammatory cytokine responses, respectively; we report GMR ratios.

To test the effect of VAS on overall cytokine responsiveness, irrespective of stimulation, a collective test for each cytokine was performed, including all conditions and adjusting for the main effect of stimulation. This, however, was only done if the test of homogeneity for the BCG effect across the different stimulations was non-significant (test of homogeneity: $P>0.05$ ) in the particular analysis, indicating that the stimulations could be merged.

Blood cell counts were analysed by linear regression on the log-transformed values, anti-logging estimates to present GMR, with CI obtained by bootstrap. Plasma levels of CRP were analysed as the frequency of individuals having a measurement $\geq 5 \mathrm{mg} / \mathrm{l}$ using Poisson regression with robust standard errors providing PR. RBP was analysed with linear regression giving a numerical difference of the mean between VAS and placebo; prevalence of $\operatorname{VAD}\left(\mathrm{RBP}<0 \cdot 83 \mu \mathrm{mol} / \mathrm{l}^{(21)}\right)$ was analysed with Poisson regression giving $\mathrm{PR}$.

Estimates were reported with $95 \%$ CI. A GMR or PR $>1$ can be interpreted as an increasing effect of VAS, whereas a GMR or $\mathrm{PR}<1$ represents a decreasing effect of VAS.

All analyses were adjusted for baseline levels and sex, as the initial randomisation was performed by sex. Children who had previously received VAS were slightly older than children not previously supplemented (data not shown). Adjusting for age in the analysis of the VAS effect stratified by previous VAS did not affect the VAS estimates (data not shown).

The estimates of the effect of VAS were stratified by sex, season of supplementation (rainy $v$. dry season) and previous VAS (yes or no); the stratification by previous VAS was further substratified by sex, as the effect modification had previously been found to be sex specific ${ }^{(14)}$.

We have corrected all estimates for multiple comparisons, separately for cytokines, leucocyte counts and CRP/RBP, using Simes false discovery rate procedure ${ }^{(22,23)}$. The statistical tests in the text are presented, both adjusted and unadjusted, whereas estimates in tables are highlighted only if significance was maintained after adjustment; $P$ values in the figures are unadjusted. 


\section{Ethical considerations}

The trial including the immunological sub-study was conducted according to the guidelines laid down in the Declaration of Helsinki, and all procedures were approved by the Ministry of Health's Committee on Research Coordination in Guinea-Bissau and by the Danish Central Ethical Committee. The trial was registered at clinicaltrials.gov (no. NCT00514891).

\section{Results}

We included 280 children, of which 258 had a blood sample taken at inclusion. At follow-up, 146 blood samples were successfully obtained and analysed (Fig. 1), giving a loss to follow-up of $43 \%$, evenly distributed between VAS and placebo arms. Of the 146 children, seventy-three had differential counts performed, 134 had RBP and CRP measured and 120 had cytokines measured. Overall, children with a successful laboratory analysis were not notably different from the children lost to follow-up with respect to background parameters, except for a larger frequency of lost males (61\% lost $v .45 \%$ followed-up) (online Supplementary Table S1). Among the analysed infants, the VAS and placebo arms were comparable with respect to background characteristics, with few exceptions: among females, children in the placebo arm were slightly taller at enrolment and their mothers were older and had larger MUAC, whereas among males fewer children in the placebo group had received OPV at birth and more had maternally reported cold (Table 1). Adjustment for these factors did not affect the estimates of VAS (data now shown).

\section{Effect of vitamin A supplementation on vitamin A status and inflammation at follow-up}

At baseline and follow-up, 70 and $72 \%$, respectively, were VAD $\left(\mathrm{RBP}<0.83 \mu \mathrm{mol} / \mathrm{l}^{(21)}\right) ; 19 \%$ had $\mathrm{CRP} \geq 5 \mathrm{mg} / \mathrm{l}$ and $14 \% \mathrm{had}$ $\mathrm{CRP} \geq 10 \mathrm{mg} / \mathrm{l}$. VAS had no effect on levels of plasma RBP or on the frequency of $\mathrm{VAD}$, overall or by sex (Table 2), and this was unaffected by vitamin A status at baseline, or by elevated CRP ( $\geq 5 \mathrm{mg} / \mathrm{l}$ ) at follow-up (data not shown). Compared with placebo, VAS significantly increased the risk of elevated CRP ( $\geq 5 \mathrm{mg} / \mathrm{l}$ ) (VAS: $28 \% v$. placebo: $12 \%$, PR: 3.15 (95\% CI 1.49, 6.63), adjusted for multiple comparisons $P=0.005$ ) (Table 2). The same conclusion was reached using a cut-off of $10 \mathrm{mg} / \mathrm{l}$ (data not shown). Sex (Table 2), season of supplementation and previous VAS (data not shown) did not significantly modify this effect.

\section{Effect of vitamin A supplementation on leucocyte count values}

Overall, there was no effect of VAS on leucocyte counts (Table 2). There was a significant interaction between VAS and sex for several subsets. In males, VAS was associated with significantly lower counts of total leucocytes, lymphocytes and monocytes, whereas the effect tended to be opposite in females (interaction between sex and VAS adjusted for multiple comparisons $P=0 \cdot 01, P=0 \cdot 04, P=0 \cdot 03$, respectively). The same

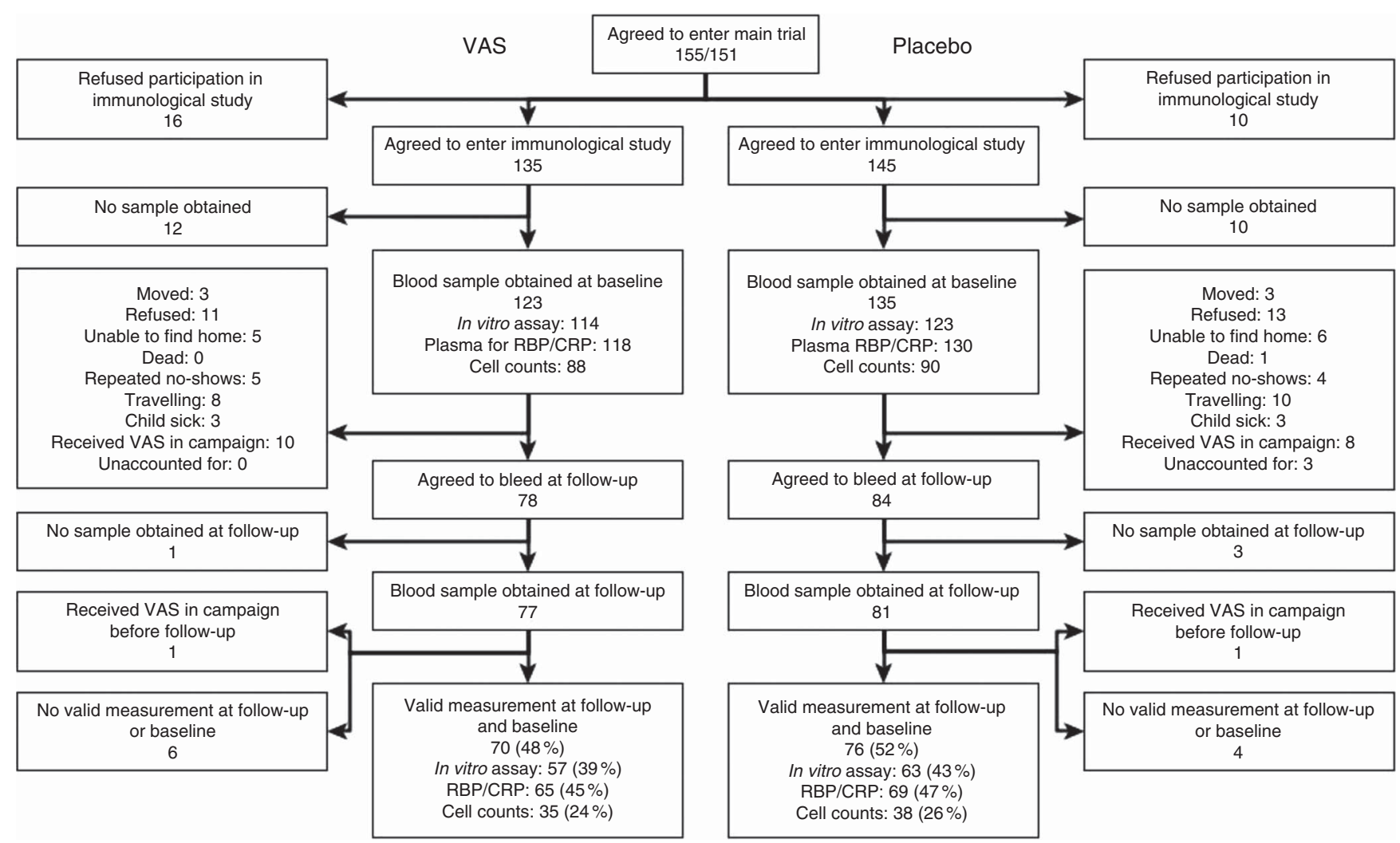

Fig. 1. Flow chart. Percentages are the fraction of total enrolled infants ( $n$ 146). VAS, vitamin A supplementation; RBP, retinol-binding protein; CRP, C-reactive protein. 
Table 1. Background characteristics at baseline*

(Percentages and numbers; medians and 10-90th percentiles; mean values and standard deviations)

\begin{tabular}{|c|c|c|c|c|c|c|c|c|c|c|c|}
\hline & \multirow[b]{4}{*}{ Background parameters } & \multicolumn{5}{|c|}{ Male } & \multicolumn{5}{|c|}{ Female } \\
\hline & & \multicolumn{2}{|c|}{ VAS } & \multicolumn{2}{|c|}{ Placebo } & \multirow[b]{3}{*}{$P$} & \multicolumn{2}{|c|}{ VAS } & \multicolumn{2}{|c|}{ Placebo } & \multirow[b]{3}{*}{$P$} \\
\hline & & \multicolumn{4}{|c|}{ Observations } & & \multicolumn{4}{|c|}{ Observations } & \\
\hline & & $\% \dagger$ & $n$ & $\% \dagger$ & $n$ & & $\% \dagger$ & $n$ & $\% \dagger$ & $n$ & \\
\hline \multirow[t]{5}{*}{ General } & All & \multicolumn{2}{|c|}{31} & \multicolumn{2}{|c|}{35} & & \multicolumn{2}{|c|}{39} & \multicolumn{2}{|c|}{41} & \\
\hline & Age (d) & \multirow{2}{*}{\multicolumn{2}{|c|}{293}} & \multirow{2}{*}{\multicolumn{2}{|c|}{292}} & 0.09 & & & & & $0 \cdot 17$ \\
\hline & Median & & & & & & \multirow{2}{*}{\multicolumn{2}{|c|}{$\begin{array}{c}288 \\
276-346\end{array}$}} & \multirow{2}{*}{\multicolumn{2}{|c|}{$\begin{array}{c}294 \\
278-375\end{array}$}} & \\
\hline & 10-90 percentile & \multicolumn{2}{|c|}{$278-364$} & \multicolumn{2}{|c|}{$276-384$} & & & & & & \\
\hline & Dry season & 45 & 14 & 49 & 17 & 0.78 & 56 & 22 & 44 & 18 & 0.26 \\
\hline \multirow{11}{*}{$\begin{array}{l}\text { Maternal and } \\
\text { socio-economic }\end{array}$} & Age of mother (years) & & & & & 0.27 & & & & & 0.005 \\
\hline & Median & & & & & & & & & & \\
\hline & 10-90 percentile & & & & & & & & & & \\
\hline & Schooling of mother (years) & & & & & 0.62 & & & & & $0 \cdot 19$ \\
\hline & Median & & & & & & & & & & \\
\hline & 10-90 percentile & & & & & & & & & & \\
\hline & MUAC of mother (mm) & & & & & 0.27 & & & & & 0.001 \\
\hline & Median & & & & & & & & & & \\
\hline & $10-90$ percentile & & & & & & & & & & \\
\hline & Electricity at home & 23 & 7 & 21 & 7 & 0.90 & 50 & 18 & 30 & 12 & 0.08 \\
\hline & Toilet inside & 10 & 3 & 12 & 4 & 0.75 & 25 & 9 & 10 & 4 & 0.08 \\
\hline Anthropometry and & Polio vaccine at birth & 84 & 26 & 57 & 20 & 0.02 & 77 & 30 & 71 & 29 & 0.53 \\
\hline & Previous VAS & 58 & 18 & 60 & 21 & 0.87 & 69 & 27 & 68 & 28 & 0.93 \\
\hline & MUAC child (mm) & & & & & 0.32 & & & & & 0.32 \\
\hline & Mean & & & & & & & & & & \\
\hline & SD & & & & & & & & & & \\
\hline & Weight (kg) & & & & & 0.31 & & & & & 0.08 \\
\hline & Mean & & 7 & & 0 & & & 3 & & 8 & \\
\hline & SD & & & & 1 & & & 0 & & 3 & \\
\hline & Height $(\mathrm{cm}) \ddagger$ & & & & & 0.98 & & & & & 0.03 \\
\hline & Mean & & & & & & & & & & \\
\hline & SD & & & & & & & & & & \\
\hline & Axillary temperature $>37^{\circ} \mathrm{C}$ & 0 & 0 & 3 & 1 & 0.34 & 0 & 0 & 0 & 0 & - \\
\hline & $\begin{array}{l}\text { Medicine use on day of enrolment } \\
\text { Maternally reported symptoms }\end{array}$ & 3 & 1 & 9 & 3 & 0.36 & 3 & 1 & 5 & 2 & 0.60 \\
\hline & Cold (runny nose) & 7 & 2 & 50 & 16 & $<0.001$ & 39 & 12 & 27 & 10 & 0.31 \\
\hline & Coughing & 29 & 9 & 43 & 15 & 0.24 & 26 & 10 & 22 & 9 & $0 \cdot 70$ \\
\hline & Diarrhoea & 10 & 3 & 0 & 0 & 0.06 & 13 & 5 & 12 & 5 & 0.93 \\
\hline & Fever & 10 & 3 & 9 & 3 & 0.91 & 8 & 3 & 5 & 2 & 0.62 \\
\hline & Vomiting & 0 & 0 & 3 & 1 & 0.336 & 5 & 2 & 0 & 0 & $0 \cdot 14$ \\
\hline & Any of the above symptoms & 42 & 13 & 60 & 21 & $0 \cdot 14$ & 54 & 21 & 44 & 18 & 0.37 \\
\hline
\end{tabular}

VAS, vitamin A supplementation; $n$, number of individuals; MUAC, mid-upper arm-circumference.

* Infants with a valid measurement of plasma biomarker, in vitro cytokines and/or differential counts at baseline and follow-up. Statistical test using $\chi^{2}$ test for categorical values;

Kruskal-Wallis test for non-normally distributed numerical values; Student's $t$ test for normally distributed numerical values.

$\dagger$ Percentage is calculated with respect to the total number of individuals with the relevant information available.

$\ddagger$ Absolute difference between VAS and placebo among females: $1.5 \mathrm{~cm}$.

pattern was observed for basophils (adjusted $P=0 \cdot 01$ ) (Fig. 2(a) and Table 2). Season did not modify the effect of VAS (data not shown). The sex-differential effects for total leucocytes, lymphocytes, monocytes and basophils were stronger in children not previously supplemented compared with those previously supplemented due to a stronger increasing effect of VAS among previously unsupplemented females than among previously supplemented females (online Supplementary Table S2).

\section{Effect of vitamin A supplementation on in vitro cytokine production, overall and by sex}

Overall, no cytokine outcomes were associated with VAS after adjustment for multiple comparisons (online Supplementary
Table S3). The effect of VAS did not depend on vitamin A status at baseline (data not shown).

There was no interaction between VAS and sex for any of the cytokine responses (Fig. 2(b)-(f)), or between VAS and season, when adjusted for multiple comparisons (data not shown).

\section{Effect of vitamin A supplementation on in vitro cytokine} production, by previous vitamin A supplementation, overall and by sex

VAS was differentially associated with several cytokine responses in children with previous VAS compared with no previous VAS (Fig. 3). For children without previous VAS, VAS was associated with a tendency towards reduced TNF- $\alpha$ 
Table 2. The effect of vitamin A supplementation (VAS) on leucocyte counts and plasma biomarkers, overall and stratified by sex (Geometric means (GM) and interquartile ranges (IQR); GM ratios (GMR) and $95 \%$ confidence intervals; difference in means (diff.) and $95 \%$ confidence intervals; proportion ratios (PR) and $95 \%$ confidence intervals)

\begin{tabular}{|c|c|c|c|c|c|c|c|c|c|c|c|}
\hline & & & \multirow{2}{*}{\multicolumn{2}{|c|}{ Placeboł }} & \multirow{2}{*}{\multicolumn{2}{|c|}{ Overall $†$}} & \multicolumn{4}{|c|}{ Sext } & \multirow[b]{3}{*}{$P \S$} \\
\hline & \multicolumn{2}{|c|}{ VAS $\ddagger$} & & & & & \multicolumn{2}{|r|}{ Male } & \multicolumn{2}{|c|}{ Female } & \\
\hline & GM & IQR & GM & IQR & GMR & $95 \% \mathrm{Cl}$ & GMR & $95 \% \mathrm{Cl}$ & GMR & $95 \% \mathrm{Cl}$ & \\
\hline & \multicolumn{2}{|c|}{$(n 35)$} & \multicolumn{2}{|c|}{$(n 38)$} & \multicolumn{2}{|r|}{$(n 73)$} & \multicolumn{2}{|r|}{ (n 39) } & \multicolumn{2}{|c|}{ (n 34) } & \\
\hline Total leucocytes & $10 \cdot 3$ & $8 \cdot 4-12 \cdot 7$ & $10 \cdot 2$ & $8 \cdot 5-11 \cdot 8$ & 0.96 & $0.84,1.09$ & $0.82 \|$ & $0.71,0.95$ & $1 \cdot 14$ & $0.92,1.43$ & $0.003 \|$ \\
\hline Lymphocytes & $5 \cdot 5$ & $4 \cdot 3-7 \cdot 0$ & $5 \cdot 5$ & $3 \cdot 8-7 \cdot 3$ & 0.91 & $0.80,1.03$ & $0.80 \|$ & $0.69,0.93$ & 1.05 & $0 \cdot 85,1 \cdot 31$ & $0.02 \|$ \\
\hline Monocytes & $1 \cdot 8$ & $1 \cdot 3-2 \cdot 3$ & $1 \cdot 8$ & $1 \cdot 3-2 \cdot 3$ & 0.99 & $0 \cdot 85,1 \cdot 16$ & $0 \cdot 81$ & $0.66,1.00$ & 1.24 & $0.93,1.64$ & $0.02 \|$ \\
\hline Neutrophils & $2 \cdot 1$ & $1 \cdot 3-2 \cdot 7$ & 1.9 & $1 \cdot 8-2 \cdot 6$ & 0.98 & $0.76,1.25$ & 0.87 & $0.65,1.17$ & $1 \cdot 11$ & $0.79,1.57$ & 0.38 \\
\hline Eosinophils & 0.3 & $0.2-0.6$ & 0.3 & $0.2-0.5$ & 0.93 & $0.67,1.28$ & 0.91 & $0.58,1.42$ & 0.96 & $0.64,1.42$ & $0 \cdot 86$ \\
\hline \multirow[t]{3}{*}{ Basophils } & 0.2 & $0 \cdot 1-0.2$ & 0.1 & $0.1-0.2$ & 0.99 & $0 \cdot 80,1 \cdot 24$ & 0.75 & $0.53,1.04$ & 1.39 & $1.08,1 \cdot 79$ & $0.004 \|$ \\
\hline & Mean & IQR & Mean & IQR & Diff. & $95 \% \mathrm{Cl}$ & Diff. & $95 \% \mathrm{Cl}$ & Diff. & $95 \% \mathrm{Cl}$ & $P \S$ \\
\hline & \multicolumn{2}{|c|}{$(n 65)$} & \multicolumn{2}{|c|}{$(n 69)$} & \multicolumn{2}{|c|}{$(n$ 134) } & \multicolumn{2}{|r|}{$(n 63)$} & \multicolumn{2}{|c|}{$(n 71)$} & \\
\hline \multirow[t]{2}{*}{$\mathrm{RBP}(\mu \mathrm{mol} / \mathrm{l})$} & 0.70 & $0.57-0.87$ & 0.73 & $0.58-0.88$ & -0.03 & $-0.10,0.04$ & 0.03 & $-0.07,0.13$ & -0.09 & $-0.18,0.01$ & 0.09 \\
\hline & \multicolumn{2}{|c|}{ Proportion } & \multicolumn{2}{|c|}{ Proportion } & PR & $95 \% \mathrm{Cl}$ & PR & $95 \% \mathrm{Cl}$ & PR & $95 \% \mathrm{Cl}$ & $P \S$ \\
\hline $\mathrm{RBP}<0.83 \mu \mathrm{mol} / \mathrm{l}$ & \multicolumn{2}{|c|}{0.72} & \multicolumn{2}{|c|}{0.72} & 1.00 & $0.81,1.23$ & 1.01 & $0.77,1.32$ & 1.00 & $0.72,1.37$ & 0.96 \\
\hline $\mathrm{CRP} \geq 5 \mathrm{mg} / \mathrm{l}$ & \multicolumn{2}{|c|}{0.28} & \multicolumn{2}{|c|}{$0 \cdot 12$} & $3 \cdot 15$ & $1.49,6.63$ & $3.65 \|$ & $1 \cdot 29,10 \cdot 33$ & $2 \cdot 79$ & $1.03,7.52$ & 0.71 \\
\hline
\end{tabular}

RBP, retinol-binding protein; CRP, C-reactive protein.

* Infants with a plasma RBP $<0.83 \mu \mathrm{mol} / \mathrm{l}$ were defined as vitamin A deficient.

† GMR or diff or PR with $95 \% \mathrm{Cl}$ comparing infants receiving VAS with infants receiving placebo. A GMR $>1$ can be interpreted as an increasing effect of VAS on the cell count.

‡ GM with IQR of leucocyte counts, or means and IQR for RBP levels, or proportion of individuals with RBP $<0.83 \mu \mathrm{mol} / \mathrm{l}$, or proportion of individuals with CRP $\geq 5 \mathrm{mg} / \mathrm{l}$ at follow-up for the placebo group and the VAS group, respectively. GM presented as $10^{6} \mathrm{cells} / \mathrm{ml}$ for all cell values.

$\S$ Statistical test for interaction between VAS and sex.

II Estimates and interaction $P$ values that are still significant after adjustment for multiple comparisons.

responses to all stimuli compared with placebo, whereas the opposite tendency was observed in children who had received VAS previously. The same pattern was also observed for IL-10 and IFN- $\gamma$ responses. Consequently, the TNF- $\alpha$ :IL-10 ratio tended to be down-regulated by VAS compared with placebo in children not previously supplemented, with the opposite tendency in children with previous VAS. A similar effect was observed for the IFN- $\gamma$ :IL-5 ratio (Fig. 3(g) and (h)).

VAS had opposite sex-differential effect directions for responses to PHA in children with $v$. without previous VAS. Among previously supplemented children, VAS tended to be associated with increased IFN $\gamma$ responses to PHA in females but decreased responses in males (interaction between VAS and sex, adjusted $P=0 \cdot 23$ ). Among children without previous VAS, VAS was associated with decreased IFN- $\gamma$ responses in females but increased responses in males (interaction between VAS and sex, adjusted $P=0 \cdot 13$ ). This resulted in a significant three-way interaction between VAS, previous VAS and sex (adjusted $P=0.0007$ ) (Fig. 2(g) and online Supplementary Table S4).

\section{Discussion}

VAS $v$. placebo given with MV from 9 months of age was associated with a number of immunological effects. First, VAS was associated with a higher proportion of children with elevated plasma CRP levels at follow-up. Second, VAS was associated with a decrease in concentrations of several blood cell subsets in males but an increase in females. Third, there was an interaction between VAS and previous VAS on several cytokine responses: VAS decreased particularly pro-inflammatory and Th1 responses in children who had not previously received VAS with an opposite effect in previously supplemented children, particularly so in females. Fourth, VAS increased PHA responses in females who had previously received VAS with an opposite effect in previously unsupplemented females; for males, this modulation of previous VAS on the effect of VAS was in the opposite direction, especially for the responses of typical lymphocyte-derived cytokines (IL-2, IL-5, IFN- $\gamma$ ).

\section{Strengths and weaknesses}

To our knowledge, the present study is the first randomised study to investigate the general (non-specific) immunological effects of VAS given with MV in a real-life setting, therefore directly testing the immunological implication of this WHO policy.

However, some limitations are noted. First, our loss to followup was higher than anticipated; many mothers refused the second blood sample. The loss to follow-up was equally distributed across the randomisation groups. Although more boys than girls were lost to follow-up, the distribution of treatment allocation among the lost was similar for boys and girls. Second, the subgroup analyses were based on small numbers, as the study was not originally designed to power the three-way interactions by sex and previous VAS. These analyses, however, were posteriorly indicated by the epidemiological study showing three-way interactions with respect to the main mortality outcome. Third, multiple testing increased the risk of chance 
(a)

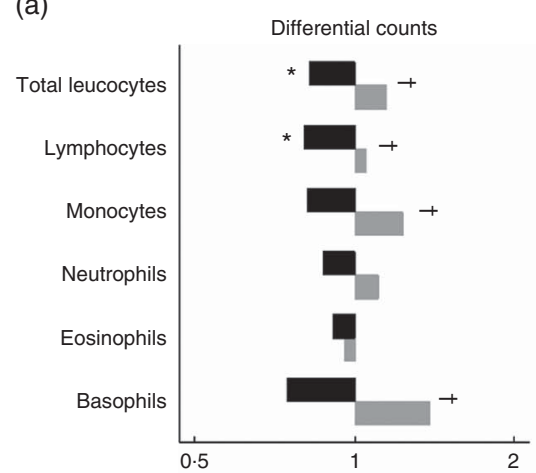

(b)

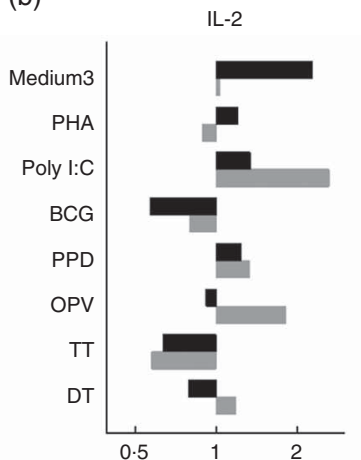

(c)

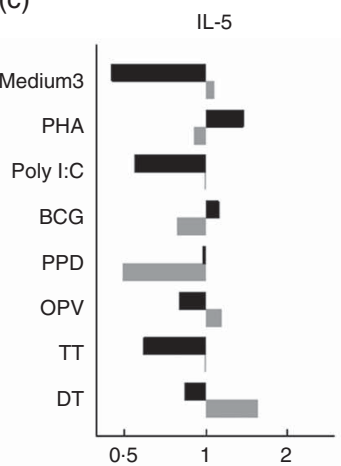

(d)

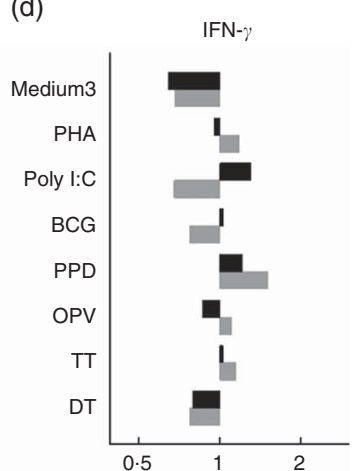

(g)

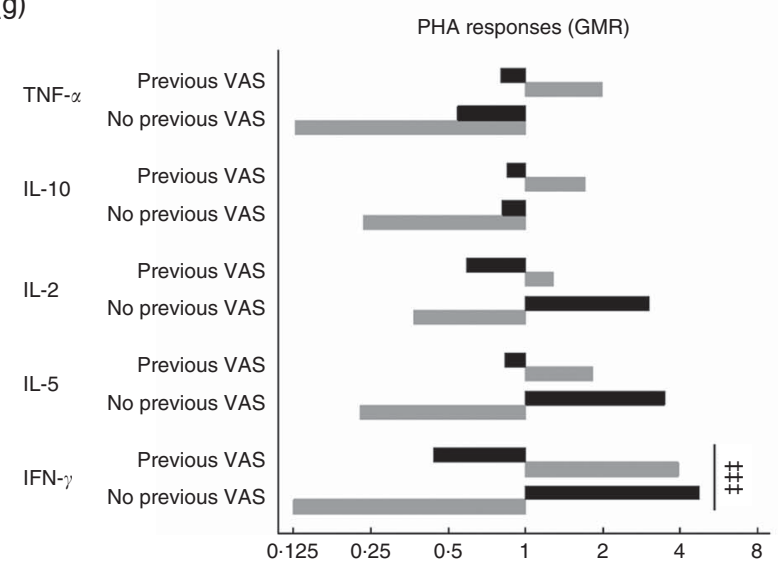

(e) (f)

Fig. 2. The effect of vitamin A supplementation (VAS) on leucocyte counts and in vitro cytokine responses. (a) Geometric mean ratios (GMR) of leucocyte counts comparing VAS with placebo, stratified by sex. (b)-(f) GMR of in vitro cytokine responses comparing VAS with placebo, stratified by sex. (g) GMR for the effect of VAS on ratios of phytohaemagglutinin (PHA) responses comparing VAS with placebo, stratified by previous VAS and sex. For TNF- $a$ and IFN- $\gamma$ responses of females with no previous VAS, the VAS effect estimates are truncated at GMR $=0.125$ for graphic reasons. Estimates were generated with linear regression for cytokines (b-g) including multiple imputations to account for measurements below the detection level. For cytokines $(\mathrm{b}-\mathrm{g})$, if more than $50 \%$ of the measurements of a given cytokine outcome was non-detectable, the estimate was obtained with Poisson regression, giving prevalence ratios. All estimates were adjusted for baseline levels. A GMR $>1$ can be interpreted as an increasing effect of VAS on the cell count. Estimates that are significant after adjustment for multiple comparisons: For VAS effect: ${ }^{*}<0.05$; for interaction between VAS and sex: $\dagger P<0.05$; for interaction between VAS, sex and previous VAS: $\ddagger \neq \ddagger P<0.001$. Medium3, culture medium only in 3-d incubation; poly I:C, polyinosine-polycytidylic acid; BCG, Bacille Calmette-Gúerin; PPD, purified protein derivative from Mycobacterium tuberculosis; OPV, oral polio vaccine, TT, tetanus toxoid; DT, diphtheria toxoid; medium1, culture medium only in 1-d incubation; LPS, lipopolysaccharide; Pam, palmitoyl(3)-cysteine-serine-lysine(4). $\square$, Male; $\square$, female.

finding. We have therefore adjusted for multiple comparisons. Fourth, previous VAS was not randomly allocated, it was provided during national community campaigns. We cannot exclude that receiving VAS in these campaigns was associated with an unmeasured health-related factor, which may in fact be the underlying effect modifier of VAS. Nonetheless, of all the recorded health-related factors, only age was associated with previous VAS, and adjustment for age in the analysis did not change the results.

\section{Comparisons with other studies}

Sex-differential effects of vitamin A supplementation. The main trial found no overall effect of VAS on mortality, but significant interaction with sex. At the 6-month follow-up, VAS increased the mortality risk in males (mortality rate ratio (MRR): 1.92; $95 \%$ CI $0.98,3.75)$ and reduced the risk in females (MRR: 0.45 (95\% CI 0.24, 0.87), interaction between VAS and sex; $P=0.003)^{(15)}$. In the present subgroup study, VAS had sex-differential effects on leucocyte counts, being associated with reduced counts in males and increased counts in females.

A few studies have investigated the effect of VAS on cell counts, even less so have taken sex into account. In line with the present findings, a previous study of VAS at birth in GuineaBissau observed that VAS resulted in a higher frequency of monocytes 6 weeks later, particularly in females, but with no significant effect in males ${ }^{(24)}$. In contrast, another study from Guinea-Bissau found no effect overall or by sex on lymphocyte counts, percentages of $\mathrm{CD}^{+}{ }^{+} \mathrm{T}$ cells, $\mathrm{CD}^{+} \mathrm{T}$ cells or the CD4: CD8 T cell ratio 3 months after VAS $v$. placebo given with MV at 6 months of age ${ }^{(25)}$.

\section{Season-differential effects of vitamin A supplementation}

The main trial found that the sex-differential effect of VAS was strongest in the dry season ${ }^{(15)}$. In the present sub-study, we found no evidence for a season-differential effect of VAS overall or by sex. 
(a)

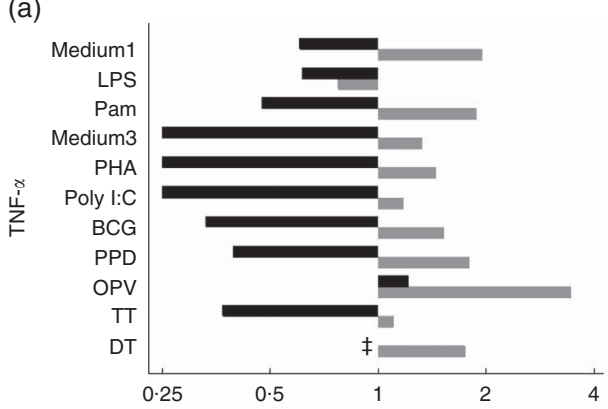

(c)

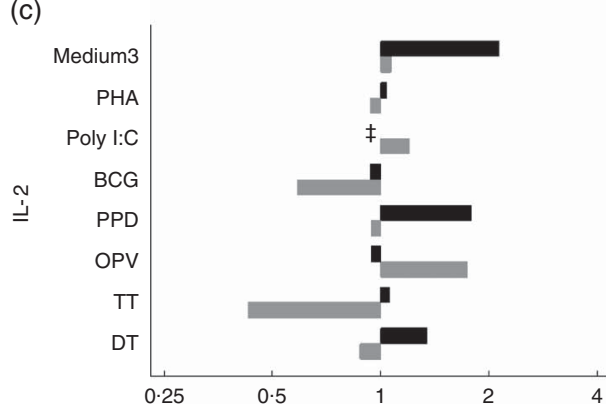

(e)

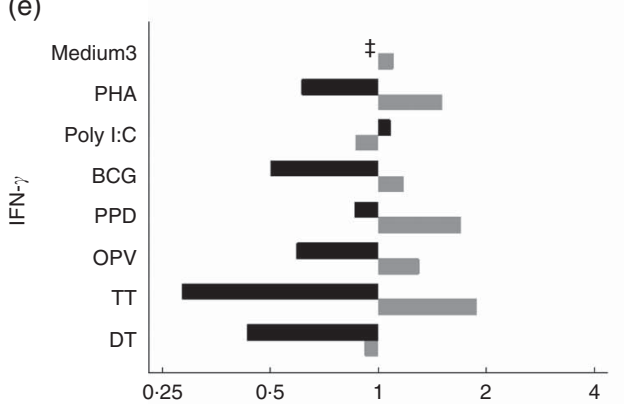

(g)

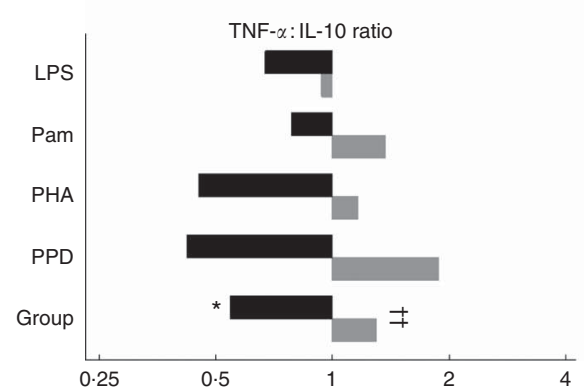

(b)

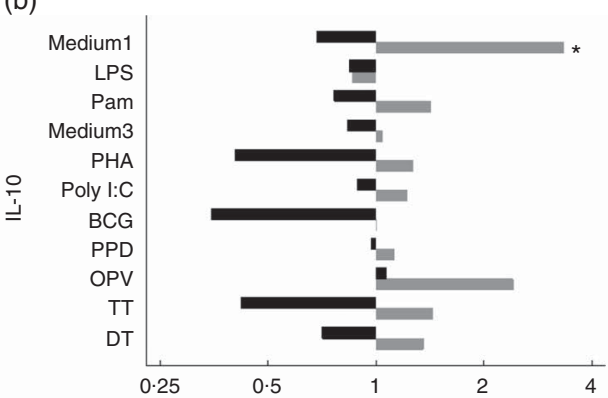

(d)

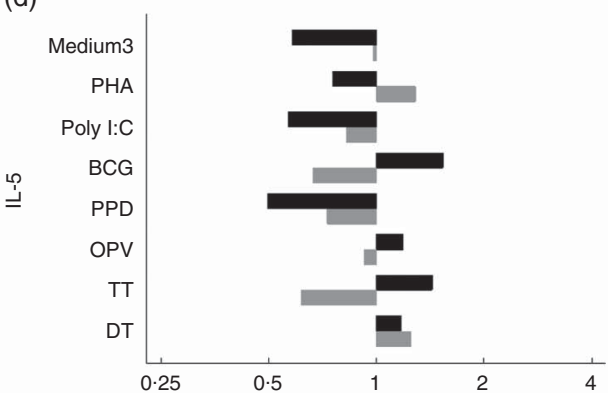

(f)

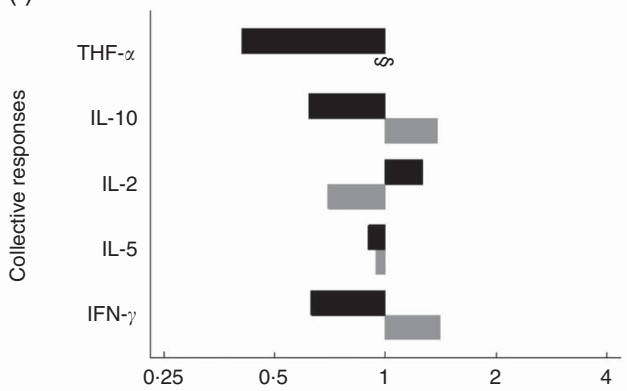

(h)

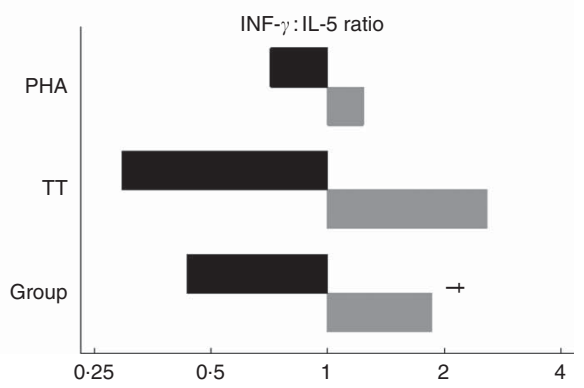

Fig. 3. The effect of vitamin A supplementation (VAS) on in vitro cytokine responses, stratified by previous VAS. Geometric mean ratios (GMR) of cytokine responses to innate agonists and vaccine antigens (a-e) for VAS compared with placebo. If $\geq 50 \%$ of the measurements of a given cytokine outcome included in the analysis was non-detectable (ND), the estimate was obtained with Poisson regression, giving prevalence ratios (PR). (f) The cytokine concentrations were analysed collectively for all stimulations in a combined analysis for each cytokine, including all stimulations and adjusting for the main effect of stimulation. Panels ( $\mathrm{g}, \mathrm{h}$ ) present GMR ratios (GMRR) for the effect of VAS on ratios of responses of TNF- $\alpha$ :IL-10 and IFN- $\gamma:$ IL-5, respectively. The analyses was only performed on distributions with $<50 \%$ ND measurements. 'Group' designates ratios of cytokine responses analysed collectively for all stimulations with $<50 \%$ ND observations. All estimates were adjusted for baseline level and sex. A GMR or GMRR (or PR) $>1$ can be interpreted as an increasing effect of VAS on the outcome. The estimates beyond the range of 0.25-4 were truncated: TNF- $a$ to medium3, TNF- $a$ to polyinosine-polycytidylic acid (Poly I:C), TNF- $a$ to Poly I:C and TNF- $a$ :IL-10 to Poly I:C in the no previous VAS stratum. $\S$ The cumulative estimate for the VAS effect on TNF- $a$ responses in previous VAS recipients could not be generated, because the estimates for the respective stimulations were too heterogeneous to be analysed collectively (test of homogeneity: $P<0.05$ ). $¥$ No estimate for the effect of VAS could be obtained for TNF- $a$ to diphtheria toxoid (DT), IL-2 to Poly I:C and IFN- $\gamma$ to medium3, due to all observations being ND at follow-up among VAS recipients (TNF- $\alpha$ to DT and IFN- $\gamma$ to medium3) or placebo recipients (IL-2 to Poly I:C), respectively, not having previously received VAS. Estimates that are significant after adjustment for multiple comparisons: ${ }^{*} P<0.05$; for interaction between VAS and previous VAS: $+P<0.05$; t $P<0.01$. Medium1, culture medium only in 1-d incubation; LPS, lipopolysaccharide; pam: palmitoyl(3)-cysteine-serine-lysine(4); medium3, culture medium only in 3-d incubation; PHA, phytohaemagglutinin; poly I:C, polyinosine-polycytidylic acid; BCG, Bacille Calmette-Gúerin; PPD, purified protein derivative from Mycobacterium tuberculosis; OPV, oral polio vaccine; TT, tetanus toxoid. , previous VAS. 
Previous vitamin A supplementation modulating the effect of vitamin A supplementation

The main trial found a strong interaction between VAS and previous VAS. In previous VAS recipients, the MRR of VAS was $5.98(1.34-26.7)$ in males and $0.18(0.05-0.62)$ in females. Among children not previously supplemented, the effects were $1.19(0.53-2.65)$ in males and $0.82(0.35-1.89)$ in females ( $P=0.007$ for three-way interaction between VAS, previous VAS and sex $)^{(15)}$. This corroborated observations from previous studies of a particularly beneficial effect of receiving repeated doses of VAS in females ${ }^{(14,26)}$.

In line with the mortality findings, in the present sub-study, the immunological effect of VAS was modulated by previous VAS, for some outcomes in a sex-differential manner. VAS tended to increase the TNF- $\alpha$ :IL- 10 ratios and IFN- $\gamma$ :IL- 5 ratios in previous VAS recipients, with opposite effects in previously unsupplemented children. To our knowledge, no other study has investigated the immunological effect of repeated dosing of VAS.

In an immunological study of the effect of MV at 4.5 months of age, a large subgroup of children had previously received VAS at birth. Exclusively among neonatal VAS recipients, MV had sex-differential effects on plasma IL-1 receptor agonist and IL-8. Furthermore, MV tended to increase the TNF- $\alpha$ :IL-10 response ratios among neonatal VAS recipients, with the opposite effect of MV in previously unsupplemented infants ${ }^{(27)}$.

These findings are in parallel with the present study, supporting differential effects of immune-modulators (be it MV or VAS) depending on previous VAS, with a dampening effect in children who receive VAS for the first time, but a larger inflammatory response in children who have previously received VAS.

The effect modulation of previous VAS on cytokine responses was furthermore sex dependent for the PHA stimulations, most significantly for IFN- $\gamma$. Of note, high IFN- $\gamma$ responses to PHA is a marker of improved survival in children ${ }^{(28,29)}$. Intriguingly, the immunological interactions for IFN- $\gamma$ to PHA were in line with the mortality findings for previously supplemented children ${ }^{(15)}$.

\section{Biological mechanisms}

Using serum RBP as indicator of vitamin A stores, many children in the present study had biochemical VAD, but VAS did not seem to affect the prevalence of VAD. A previous study from Guinea-Bissau examined the effect of VAS at birth on vitamin A status at age 6 weeks and at age 4 months and also found no measurable effect on vitamin A status ${ }^{(21)}$, but in line with the present trial there were, nonetheless, measureable effects on mortality $^{(13)}$ and immunological outcomes ${ }^{(24)}$. Of note, a biological model of vitamin A uptake and catabolism in children has predicted a return of serum retinol levels to baseline only 2 months after high-dose $\mathrm{VAS}^{(30)}$. These findings may suggest that changes in vitamin A status measured as blood biochemical markers do not serve as a good predictor of VAS effects on mortality or on immune function. In other words, the effect of VAS seems not to be mediated via an effect on vitamin A status, but rather through immunomodulation. Other analyses such as tissue examination may be more sensitive indicators of vitamin A status than blood biomarkers. This, however, is not feasible in population studies such as the present; in order to categorise children as deficient or not, serum biomarkers can indeed be used ${ }^{(31)}$.

Most immunological in vitro studies assign anti-inflammatory capacities to retinoid acids, metabolites of vitamin $\mathrm{A}^{(32-36)}$. Most recently, it has been shown that pre-incubation of human monocytes with all-trans retinoic acid (ATRA) reduces the secondary inflammatory responses to innate agonist stimulation in an ATRA dose-dependent manner. Moreover, ATRA suppressed the increasing effect on BCG priming of monocytes in the secondary responses. The suppressive effect of ATRA was observed at the epigenetic level by an increase in suppressive methylation markers at the IL- 6 and TNF- $\alpha$ promoters, which lasted at least $10 \mathrm{~d}$ in the in vitro model ${ }^{(37)}$. The dampening effect in vitro of vitamin A on cytokine responses is in concordance with the present in vivo findings in children not previously supplemented.

In conclusion, the present study supports that VAS has immune-modulating effects and that the effects may depend on the sex of the recipient. The immunological effects of VAS may be modified by previous VAS, corroborating findings from epidemiological studies indicating a long-lasting imprinting effect of VAS on the immune system.

\section{Acknowledgements}

The authors acknowledge the essential contribution by Mathias Jul Jørgensen, who was responsible for sample collection and partook in the sample analysis, but who has other obligations and has not wished to co-author this paper. The authors furthermore thank Professor Mogens Helweg Claesson for expert advice on the design of the study and Jürgen Erhardt for his help with establishing the RBP ELISA assay.

The main support came from an ERC Starting Grant to CSB (grant ERC-2009-StG-243149). The study was furthermore made possible through the financial support from the Augustinus Foundation, A. P. Møller og Hustru Christine Mc-Kinney Møllers fond til almene Formaal, Dagmar Marshall foundation, the Lundbeck foundation and the Danish Agency for Science, Technology and Innovation. Purchase of the ABX Pentra was funded by A. P. Møller og Hustru Christine Mc-Kinney Møllers fond til almene Formaal. P. A. holds a research professorship grant from the Novo Nordisk Foundation. Research Center for Vitamins and Vaccines is supported by Danish Research Foundation (grant number DNRF108). The funding bodies had no role in the study design and conduct or in data analysis and drafting of the manuscript.

C. S. B., P. A., A. B. F. conceived the idea for the study; C. S. B., C. E., E. S., M. Y. designed the experiments; A. B. F. supervised the collection of samples and field data; E. S. performed the laboratory analyses; K. J. J. analysed the data; A. A. supervised the statistical analyses; K. J. J. wrote the first draft of the manuscript; all the authors read and approved the final version of the manuscript.

The authors declare that there are no conflicts of interest. 


\section{Supplementary material}

For supplementary material/s referred to in this article, please visit http: //dx.doi.org/doi:10.1017/S0007114515004869

\section{References}

1. Raverdeau M \& Mills KH (2014) Modulation of $T$ cell and innate immune responses by retinoic acid. J Immunol 192, 2953-2958.

2. World Health Organization (2009) Global Prevalence of Vitamin A Deficiency in Populations at Risk 1995-2005. WHO Global Database on Vitamin A Deficiency. Geneva: WHO.

3. World Health Organization (2011) Guideline: Vitamin A Supplementation in Infants and Children 6-59 Months of Age. Geneva: WHO.

4. World Health Organization (2014) WHO recommendations for routine immunization - summary tables. http://www.who. int/immunization/policy/immunization_tables/en/ (accessed May 2014).

5. Benn CS, Netea MG, Selin LK, et al. (2013) A small jab - a big effect: nonspecific immunomodulation by vaccines. Trends Immunol 34, 431-439.

6. Aaby P, Martins CL, Garly ML, et al. (2010) Non-specific effects of standard measles vaccine at 4.5 and 9 months of age on childhood mortality: randomised controlled trial. BMJ 341, c6495.

7. Shann F (2013) Nonspecific effects of vaccines and the reduction of mortality in children. Clin Ther 35, 109-114.

8. Benn CS, Balde A, George E, et al. (2002) Effect of vitamin A supplementation on measles-specific antibody levels in Guinea-Bissau. Lancet 359, 1313-1314.

9. Benn CS, Aaby P, Bale C, et al. (1997) Randomised trial of effect of vitamin A supplementation on antibody response to measles vaccine in Guinea-Bissau, west Africa. Lancet 350 , 101-105.

10. Benn CS, Bale C, Sommerfelt H, et al. (2003) Hypothesis: vitamin A supplementation and childhood mortality: amplification of the non-specific effects of vaccines? Int J Epidemiol 32, 822-828.

11. Benn CS, Fisker AB, Napirna BM, et al. (2010) Vitamin A supplementation and BCG vaccination at birth in low birthweight neonates: two by two factorial randomised controlled trial. BMJ 340, c1101.

12. Benn CS, Rodrigues A, Yazdanbakhsh M, et al. (2009) The effect of high-dose vitamin A supplementation administered with BCG vaccine at birth may be modified by subsequent DTP vaccination. Vaccine 27, 2891-2898.

13. Benn CS, Diness BR, Roth A, et al. (2008) Effect of 50000 IU vitamin A given with BCG vaccine on mortality in infants in Guinea-Bissau: randomised placebo controlled trial. BMJ 336, 1416-1420.

14. Fisker AB, Aaby P, Rodrigues A, et al. (2011) Vitamin A supplementation at birth might prime the response to subsequent vitamin A supplements in girls. Three year follow-up of a randomized trial. PLOS ONE 6, e23265.

15. Fisker AB, Bale $\mathrm{C}$, Rodrigues $\mathrm{A}$, et al. (2014) High-dose vitamin A with vaccination after 6 months of age: a randomized trial. Pediatrics 134, e739-e748.

16. World Health Organization (1997) Vitamin A Supplements: A Guide to Their Use in the Treatment of Vitamin A Deficiency and Xerophthalmia, 2nd ed. Geneva: WHO.

17. Erhardt JG, Estes JE, Pfeiffer CM, et al. (2004) Combined measurement of ferritin, soluble transferrin receptor, retinol binding protein, and C-reactive protein by an inexpensive, sensitive, and simple sandwich enzyme-linked immunosorbent assay technique. J Nutr 134, 3127-3132.
18. Uh HW, Hartgers FC, Yazdanbakhsh M, et al. (2008) Evaluation of regression methods when immunological measurements are constrained by detection limits. BMC Immunol 9, 59.

19. Andersen A, Benn CS, Jorgensen MJ, et al. (2013) Censored correlated cytokine concentrations: multivariate Tobit regression using clustered variance estimation. Stat Med 32, 2859-2874.

20. Royston P (2007) Profile likelihood for estimation and confidence intervals. Stata J 7, 376-387.

21. Fisker AB, Lisse IM, Aaby P, et al. (2007) Effect of vitamin A supplementation with BCG vaccine at birth on vitamin A status at 6 wk and 4 mo of age. Am J Clin Nutr 86, 1032-1039.

22. Newson R (2003) Multiple-test procedures and smile plots. Stata J 3, 109-132.

23. Benjamini Y \& Yekutieli D (2001) The control of the false discovery rate in multiple testing under dependency. Ann Stat 29, 1165-1188.

24. Jorgensen MJ, Fisker AB, Sartono E, et al. (2013) The effect of at-birth vitamin A supplementation on differential leucocyte counts and in vitro cytokine production: an immunological study nested within a randomised trial in Guinea-Bissau. Br J Nutr 109, 467-477.

25. Benn CS, Lisse IM, Bale C, et al. (2000) No strong long-term effect of vitamin A supplementation in infancy on CD4 and CD8 T-cell subsets. A community study from Guinea-Bissau, West Africa. Ann Trop Paediatr 20, 259-264.

26. Fisker AB, Aaby P, Bale C, et al. (2012) Does the effect of vitamin A supplements depend on vaccination status? An observational study from Guinea-Bissau. BMJ Open 2, e000448.

27. Jensen KJ, Sondergaard M, Andersen A, et al. (2014) A randomized trial of an early measles vaccine at 4(1/2) months of age in Guinea-Bissau: sex-differential immunological effects. PLOS ONE 9, e97536.

28. Rose MV, Kimaro G, Nissen TN, et al. (2012) QuantiFERON ${ }^{\circledR}$ TB gold in-tube performance for diagnosing active tuberculosis in children and adults in a high burden setting. PLOS ONE 7, e37851.

29. Andersen A, Jensen KJ, Erikstrup C, et al. (2014) Both very low- and very high in vitro cytokine responses were associated with infant death in low-birth-weight children from Guinea Bissau. PLOS ONE 9, e93562.

30. Allen LH \& Haskell M (2002) Estimating the potential for vitamin A toxicity in women and young children. J Nutr $\mathbf{1 3 2}$, 2907S-2919S.

31. Tanumihardjo SA (2012) Biomarkers of vitamin A status: what do they mean? In World Health Organization. Report: Priorities in the Assessment of Vitamin A and Iron Status in Populations, Panama City, Panama, 15-17 September 2010. Geneva: WHO.

32. Dheen ST, Jun Y, Yan Z, et al. (2005) Retinoic acid inhibits expression of TNF-alpha and iNOS in activated rat microglia. Glia 50, 21-31.

33. Mathew JS \& Sharma RP (2000) Effect of all-trans-retinoic acid on cytokine production in a murine macrophage cell line. Int J Immunopharmacol 22, 693-706.

34. Mehta K, McQueen T, Tucker S, et al. (1994) Inhibition by all-trans-retinoic acid of tumor necrosis factor and nitric oxide production by peritoneal macrophages. J Leukoc Biol $\mathbf{5 5}$, 336-342.

35. Mucida D, Park Y \& Cheroutre H (2009) From the diet to the nucleus: vitamin A and TGF-beta join efforts at the mucosal interface of the intestine. Semin Immunol 21, 14-21.

36. Wojtal KA, Wolfram L, Frey-Wagner I, et al. (2013) The effects of vitamin A on cells of innate immunity in vitro. Toxicol In Vitro 27, 1525-1532.

37. Arts RJ, Blok BA, van Crevel R, et al. (2015) Vitamin A induces inhibitory histone methylation modifications and down-regulates trained immunity in human monocytes. J Leukoc Biol 98, 129-136. 